\title{
Lipid Emulsion May Augment Early Blood Pressure Recovery in a Rabbit Model of Atenolol Toxicity
}

Dear Editor,

Lipid emulsion has been identified as a potential antidote in the treatment of poisoning with drugs of high lipophilicity [1]. Both pharmacokinetic and pharmacodynamic mechanisms have been forwarded to explain this therapeutic effect. One explanation is that lipophilic drug sequestration within intravascular lipid droplets may decrease free plasma concentration and thus target organ tissue levels. Alternatively, bolus administration of free fatty acids may augment cardiac performance via overcoming pharmacologically induced impediments to myocardial oxidative phosphorylation, or through elevation of intramyocyte calcium concentration [1].

Demonstration of effect for lipid emulsion in a hydrophilic drug toxidrome would lend support for a metabolic explanation of lipid's effects. Atenolol is a hydrophilic beta-blocker with a water:octanol coefficient of 0.23 . We determined to explore the hemodynamic response to lipid resuscitation from atenololinduced hypotension in a previously established intact rabbit model of drug-induced hypotension $[2,3]$.

This study was undertaken at the Ruakura Agresearch facility, Hamilton New Zealand, and was approved by the Ruakura Animal Ethics Committee. The conditions of rabbit husbandry in addition to sedation, instrumentation, and mechanical ventilation protocols were identical to our previously published work [3]. The study was $80 \%$ powered at the 0.05 significance level for a 1.5 standard deviation (approximately $10 \mathrm{mmHg}$ [2]) in blood pressure with 10 subjects in each group.

Following preliminary dose ranging experiments (6 animals), atenolol $25 \mathrm{mg} / \mathrm{mL}$ was infused at $1 \mathrm{~mL} / \mathrm{min}$ to a target mean arterial pressure (MAP) of $60 \%$ baseline. In a number of subjects however reduction in MAP with atenolol infusion was precipitous following a prolonged period of relative stability, with irreversible cardiovascular collapse seen prior to stabilization at the intended toxic endpoint. At toxicity, 6-mL/kg 20\% Intralipid or 6-mL/kg $0.9 \%$ saline solution was infused over a 4 -minute period, in line with our previous work. Comparison of hemodynamic metrics was conducted at baseline, toxicity, immediately postresuscitation, and 15 minutes postresuscitation.

Statistical analysis was undertaken using Graphpad InStat(3.06) software (GraphPad Software Inc, La Jolla, CA 92037). Fischer's exact and Mann Whitney tests were used as appropriate. Statistical significance set at the 0.05 level.

Six of 10 subjects survived to stabilization at the predefined point of toxicity in the lipid group, with 8 surviving to this point

\section{Table 1: Subject Baseline Characteristics, MAP} Throughout Study Protocol*

\begin{tabular}{lcc}
\hline & Lipid group & Saline group \\
\hline Weight (g) & $2615(2600-2900)$ & $2779(2593-2900)$ \\
\hline Age (days) & $95(92-95)$ & $95(95-97)$ \\
\hline Atenolol (mg) & $562(334-870)$ & $660(345-1080)$ \\
\hline Sex (Male:Female) & $3: 3$ & $4: 4$ \\
\hline Baseline MAP (mmHg) & $73(67-77)$ & $65(59-70)$ \\
\hline Toxicity MAP & $44(34-50)$ & $37(34-43)$ \\
\hline Postresuscitation MAP & $51(41-56)$ & $34(31-45)$ \\
\hline 15-minute MAP & $42(38-45)$ & $51(34-63)$ \\
\hline *All data presented as median (interquartile range) & \\
\hline
\end{tabular}

in the saline group. All animals subsequently survived to the time-point immediately postresuscitation. Two saline-treated subjects died in the 15 minutes postresuscitation, with no further subjects dying in the lipid group $(p=0.48)$. Baseline animal characteristic and MAP are shown in Table 1.

No statistically significant difference was observed for MAP or pulse rate at any time between groups. A trend toward greater MAP in the lipid-treated group immediately postresuscitation approached significance $(p=0.10)$. The observed increment in MAP following resuscitation was greater in the lipid-treated group (Figure 1).

Early recovery from atenolol toxicity may have been augmented by lipid resuscitation in this model with the change in blood pressure immediately postresuscitation greater in the lipid group. This finding lends inferential support to a metabolic explanation for the effects of lipid in beta-blocker toxicity. Beta-blockers are known to cause inhibition of mitochondrial oxidative phosphorylation [4]. It is conceivable that lipid could serve to reverse this inhibition through an effect of mass action on mitochondrial free fatty acid provision, or alternatively through increasing intramyocyte calcium.

Methodologic limitations mitigate against clearly attributing the observed association to lipid. Although absolute reduction in median MAP during atenolol infusion was equivalent between groups, proportionate reduction was greater in the saline group. It is therefore possible that members of the saline group were 


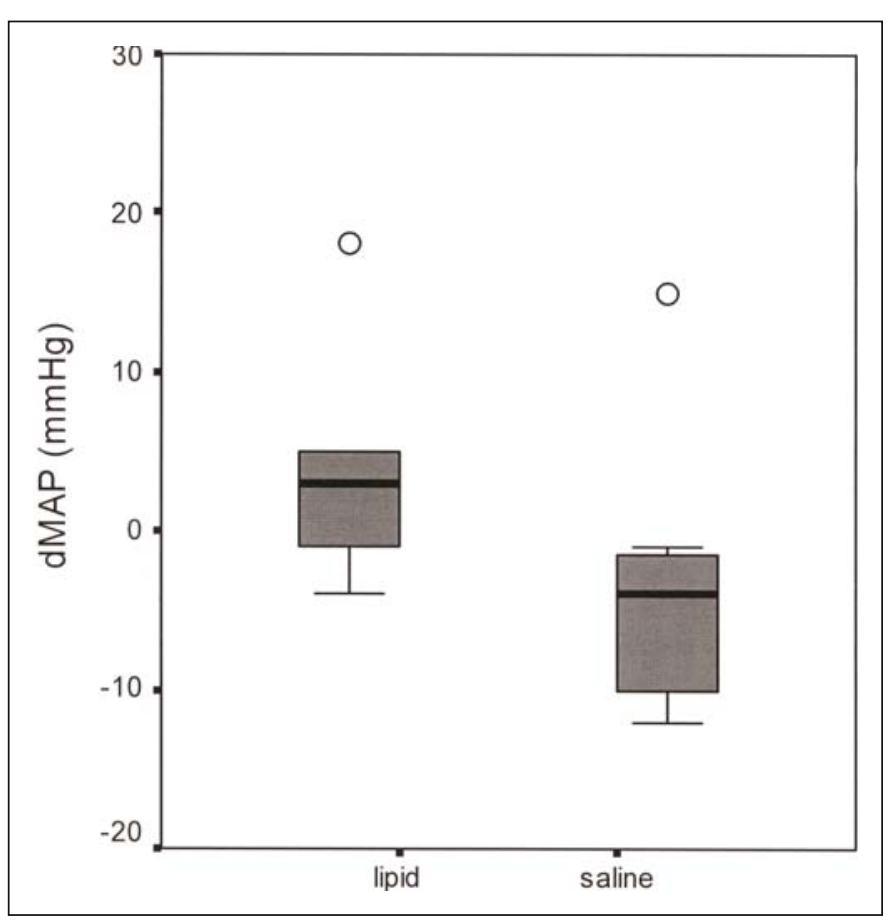

Figure 1: Change in MAP (dMAP) immediately following resuscitation $(0$, outlier; $p=0.02)$.

more severely poisoned and simply took longer to recover. In addition, more subjects in the lipid group died before stabilization at the point of toxicity, thereby potentially selecting more robust animals, with greater likelihood of recovery regardless of treatment.

Change in blood pressure immediately postlipid resuscitation was a post hoc endpoint, and thus establishes the need for further study, rather than proving association. It does establish the hypothesis that lipid emulsions may be effective in hydrophilic drug cardiotoxicity as one worthy of further pursuit, however. The present study also suggests that a more stable pharmacological model, such as esmolol-induced shock [5], would prove more suitable for future study.

We conclude that early recovery from atenolol toxicity may have been augmented by lipid resuscitation in this model. Further work investigating utility of lipid resuscitation via a metabolic effect in hydrophilic drug cardiotoxicity is warranted.
Dr. Grant Cave (FACEM)

Hutt Hospital, Lower Hutt, New Zealand

University of Otago, Wellington Medical School, Wellington,

New Zealand

grantcave@gmail.com

c/o Emergency Department, Hutt Hospital

High Street, Lower Hutt

Private Bag 31-907

Lower Hutt

5040

New Zealand

Dr. Martyn Harvey (FACEM)

Department of Emergency Medicine, Waikato Hospital

Pembroke Street, Hamilton, New Zealand

harveym@waikatodhb.govt.nz

c/o Emergency Department, Waikato Hospital

Pembroke Street

Private Bag 3200

Hamilton 3240

New Zealand

This study was funded by a grant from the Morson Taylor Research Award.

The authors have no potential financial conflicts of interest to report.

\section{REFERENCES}

1. Turner-Lawrence D, Kerns W. Intravenous fat emulsion: a potential novel antidote. J Med Toxicol 2008;4:109-114.

2. Harvey M, Cave G. Intralipid outperforms sodium bicarbonate in a rabbit model of clomipramine toxicity. Ann Emerg Med 2007;49:178-185.

3. Harvey M, Cave G. Intralipid infusion ameliorates propranolol induced hypotension in rabbits. J Med Toxicol 2008;4:71-76.

4. Dreisbach A, Greif R, Lorenzo B, et al. Lipophilic BetaBlockers Inhibit Rat Skeletal Muscle Mitochondrial Respiration. Pharmacology 1993;47:295-299.

5. Sasao J, Tarver S, Kindsher J, et al. In rabbits, landiolol, a new ultra short acting beta blocker, exerts a more potent negative chronotropic effect and less effect on blood pressure than esmolol. Can J Anaesth 2001; 41:985-989. 